\title{
Electron-beam writing of photonic crystal patterns using a large beam-spot diameter
}

\author{
S Kim, H Chong, R M De La Rue, J H Marsh and A C Bryce \\ Department of Electronics and Electrical Engineering, University of Glasgow, \\ Glasgow G20 8LT, UK \\ E-mail: s.kim@elec.gla.ac.uk
}

Received 10 March 2003, in final form 8 July 2003

Published 11 August 2003

Online at stacks.iop.org/Nano/14/1004

\begin{abstract}
A simple and cost-effective method of patterning photonic crystal $(\mathrm{PhC})$ or similar structures by electron beam lithography is demonstrated. Instead of using fine resolution and a multitude of small-sized beam spots to write a single hole, the combination of a single electron-beam spot having the size of the hole and a position resolution equal to the period of the $\mathrm{PhC}$ is used. This single-spot exposure scheme typically shows an order-of-magnitude reduction in the required job time compared to that for the conventional multi-spot exposure scheme. In addition, the inherently circular shape of the electron-beam spot is directly transferred into the UV3 resist used in this work, leaving uniform circular holes after development. A cross-sectional view of the holes shows a very good verticality in the sidewalls of the resist. A modified scheme using four spots is also demonstrated which alleviates some limitations encountered in the single-spot exposure scheme.
\end{abstract}

\section{Introduction}

Photonic crystals (PhCs) have been widely used in the design and realization of a variety of optoelectronic devices such as micro-cavity lasers and channel waveguides [1-5]. While much of the research activity has been focused on discrete devices, one of the important current trends is toward the monolithic integration of many elements to achieve more complex functionality [6]. As the demand for large-area production and complex $\mathrm{PhC}$-type structures grows, there is a need for an efficient and simple method of generating PhCs. As one alternative to direct-write electron beam lithography (EBL), interferometric or holographic lithography has been successfully used to fabricate $\mathrm{PhC}$ structures [79]. This technique can be useful for large-area patterning of simple $\mathrm{PhC}$ structures, but is not suitable for integration of complex structures such as waveguide bends, coupled cavities and waveguides. Another alternative approach, using deep UV lithography, has recently been demonstrated [10]. As this technique is an optical lithography technology based on excimer lasers at wavelengths of $248 \mathrm{~nm}$ or shorter, it can be very useful for mass production of PhCs. But the technique requires the extra step of mask generation, as compared to the direct-write approach, and optical proximity correction is necessary at the mask design stage. At present, no available technology compares with EBL in its flexibility for the generation of complex patterns and possibilities for feature sizes below $100 \mathrm{~nm}$. Thus an improved EBL scheme for $\mathrm{PhC}$ patterns clearly merits investigation. Our work has been carried out on a Leica Beamwriter EBPG5 and hence we shall merely refer to the machine as a Beamwriter in the rest of this paper.

The conventional approach to direct electron-beam writing of $\mathrm{PhC}$ patterns is firstly to define a single hole at the design stage. A regular polygon is normally used as an approximation to a circle. A two-dimensional array of the polygons is then generated to form a $\mathrm{PhC}$ pattern. If the pattern to be written is large, the arrays are usually divided into hierarchies of sub-cells to make it easier for the Beamwriter to handle the file. The job time for beam-writing of $\mathrm{PhC}$ patterns can be extremely long since multiple electron-beam spots with fine resolution (that is, the writing grid) are used to define a single hole. The job time can mount further when the area to be written is large. In addition, an increase in the number of arc points for the polygon to better approximate a circular hole results in a larger file size and consequently an increase in the job time.

In this paper, we describe a simple technique of directwrite EBL of PhC patterns which drastically reduces the job time and thus is cost-effective. The idea is to use a single 


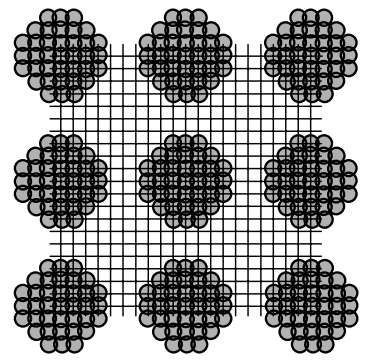

(a)

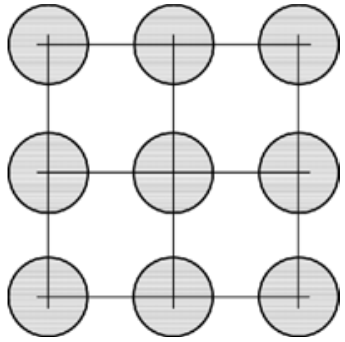

(b)
Figure 1. Schematic diagrams of electron-beam writing of $\mathrm{PhC}$ patterns: (a) by smaller-size multiple-spot exposure, (b) larger-size single-spot exposure. Each grid represents the resolution unit.

electron-beam spot with a larger spot size, rather than multiple spots with a smaller spot size, to define a single hole. The spot size (or diameter) of the beam is defined to be, if a Gaussian beam profile is assumed, a diameter which contains half the total beam current and where the local current density is halfmaximum. A square lattice becomes a natural choice for this purpose, as the Beamwriter itself uses a square-lattice scan system. Square-lattice $\mathrm{PhCs}$ have not been investigated as much as triangular lattice $\mathrm{PhCs}$ due to their smaller band gap for TE-polarization. However, as there are on-going efforts to better understand and utilize square-lattice PhCs, this area is an open possibility for device applications [11-16].

Details of how to design PhC patterns by the single-spot exposure scheme will be described and results are shown in section 2. A comparison in job time has also been made between single-spot and multiple-spot exposure schemes. In section 3, some limitations encountered in the single-spot exposure scheme are discussed and another modified exposure scheme using four large spots together with a small resolution is described, as well as some results. Finally, conclusions are given in section 4 .

\section{Single-spot exposure scheme}

The idea of using single-spot exposure has been reported previously, e.g. by Wendt et al [17]. However, the process reported had limited success and the full potential of this approach was not investigated. Moreover, the results obtained in terms of the eventual structure produced were limited. As Wendt et al used a $6 \mathrm{~nm}$ beam-spot diameter together with a $5 \mathrm{~nm}$ resolution, the holes were too small, nominally $50 \mathrm{~nm}$ in diameter, to be used as a suitable etch mask. Our approach is significantly different in the sense that a substantially larger electron-beam spot (112 $\mathrm{nm}$ in diameter) is used, as well as coarser resolution $(300 \mathrm{~nm})$. Figure 1 shows schematically how a single hole is defined in the conventional exposure scheme and in our single-spot exposure scheme. In the conventional method, the spot size employed is almost ten times smaller than the desired hole size in order to obtain a well-defined hole. In our exposure scheme, a spot comparable to the size of the hole to be defined, and a resolution equal to the period of the PhC array, are used. Designing PhC structures with complex patterns is straightforward. In WaveMaker, the commercially supplied design software that has been used, a

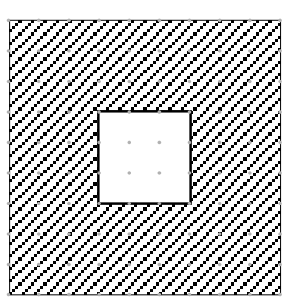

(a)

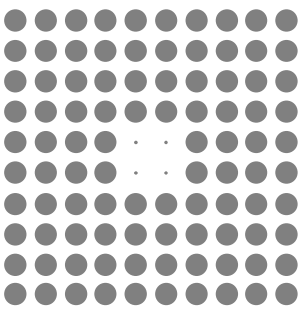

(b)
Figure 2. (a) Area definition in CAD software, (b) generated patterns after single-spot electron-beam exposure. The distance between the small dots (which is the same as the hole centre-to-centre spacing) corresponds to the resolution of the CAD pattern, and consequently the period of holes in the final beam-written pattern. Large circles represent single electron-beam spots.

pattern is defined, as shown in figure 2(a), using a resolution which is the same as the periodicity of the hole pattern to be written. When the Beamwriter scans the pattern it will expose each pixel within the shaded area with a single electron-beam spot. If a positive resist is used, the resulting pattern after development, as shown in figure 2(b), will have a periodically placed series of holes within the original pattern.

A test run was carried out to check the scheme. Test patterns were designed with a $300 \mathrm{~nm}$ resolution. A $200 \mathrm{~nm}$ $\mathrm{SiO}_{2}$ layer was deposited by PECVD (plasma enhanced chemical vapour deposition) on top of a GaAs substrate to help with adhesion of the resist to the sample. The substrate was cleaned and then a layer of standard commercial primer was spun onto the sample before a single layer of UV3 positive resist was spun onto the substrate. The sample was then heated to $130^{\circ} \mathrm{C}$ on a hotplate for $1 \mathrm{~min}$ to cure the resist. Beam writing was carried out using a nominally $112 \mathrm{~nm}$ electronbeam spot diameter and a $300 \mathrm{~nm}$ resolution. Doses varied from 10 to $100 \mu \mathrm{C} \mathrm{cm}^{-2}$. The electron-beam energy was $50 \mathrm{kV}$ and the sample was developed in CD26 developer after a post-exposure-bake at $135^{\circ} \mathrm{C}$ for $1 \mathrm{~min}$.

The developed patterns were inspected in a scanning electron microscope (SEM). Figure 3 shows a top view of the pattern processed at a dose of $10 \mu \mathrm{C} \mathrm{cm}^{-2}$. The resulting hole diameter is $170 \mathrm{~nm}$. The circularity and the uniformity of holes are clearly both very good. In addition, as can be seen in figure 4, good vertical profiles are achieved in the resist, which is an important issue for use of the resist pattern as a dry-etch mask. The holes are fully developed to the bottom and the layer beneath the holes is a $\mathrm{SiO}_{2}$ layer. Overall, the structural properties of our single-spot $\mathrm{PhCs}$ seem to be, at the least, comparable in quality to those of $\mathrm{PhC}$ patterns written with a multi-spot scheme. Though there can be concerns about the reproducible definition of specific hole sizes in this single-spot exposure scheme, because the resist is not receiving a uniform dose across the entire hole, we could produce almost same hole sizes repeatedly through a tight control of the development. However, the reproducibility of the process on a variety of resists is deemed worthy of investigation.

Implementing complex patterns such as the one shown in figure 5 is much easier with a single-spot exposure scheme. 


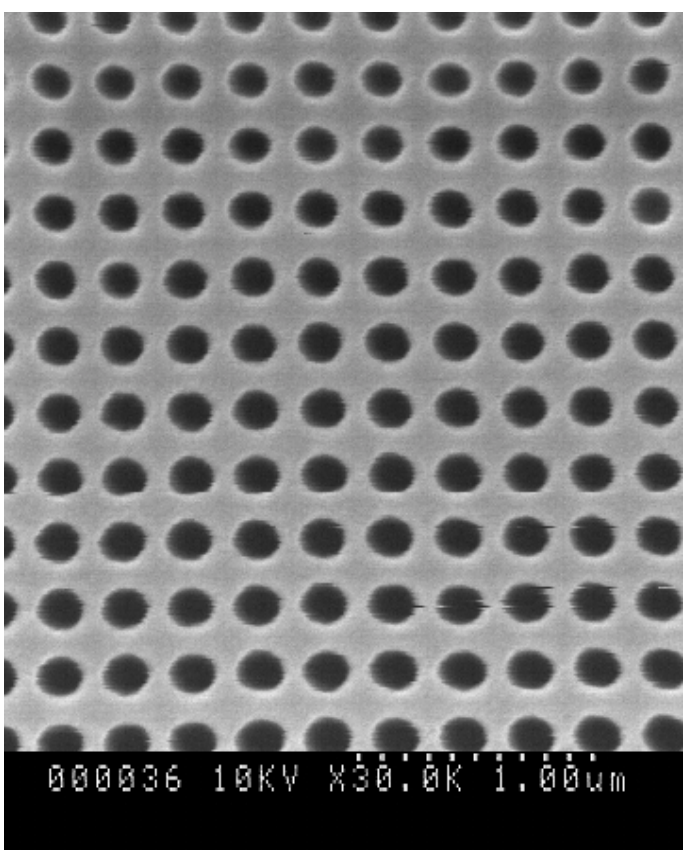

Figure 3. SEM image of $\mathrm{PhC}$ patterns on UV3 resist. Each hole was exposed to a single electron-beam spot with $112 \mathrm{~nm}$ spot size and $10 \mu \mathrm{C} \mathrm{cm}^{-2}$ dose.

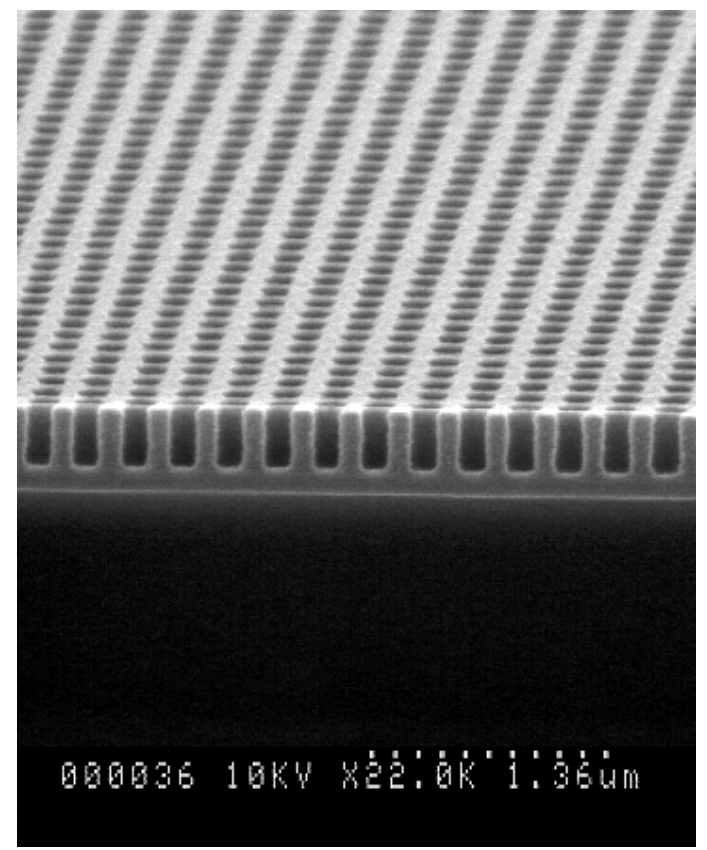

Figure 4. Cross-sectional image of the sample shown in figure 3. The UV3 resist is fully developed and the underlying layer is a $200 \mathrm{~nm} \mathrm{SiO}{ }_{2}$ layer. Good verticality was achieved in the sidewalls.

At the design stage, it is only necessary to define areas where holes are to be written instead of placing holes at desired points. Another vitally important merit of our technique is that the job time (that is, the beam-writing time) can be drastically reduced compared to a typical multi-spot exposure process. Figure 6 shows the estimated job times, given by the electron-beam software prior to the actual beam-writing, in both schemes

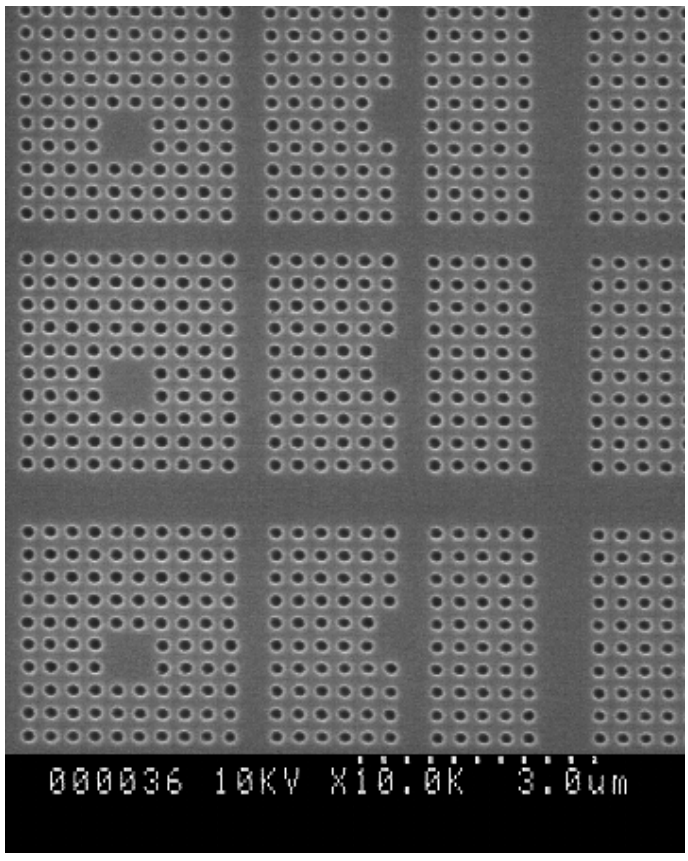

Figure 5. SEM image of a test pattern with $\mathrm{PhC}$ channel waveguides and resonator structures written by the single-spot exposure scheme.

to write PhC patterns with a period of $0.3 \mu \mathrm{m}$ in an area of $500 \times 500 \mu \mathrm{m}^{2}$. The dose was held constant at $30 \mu \mathrm{C} \mathrm{cm}^{-2}$. The resolution and the spot size for the multi-spot exposure scheme were 5 and $12 \mathrm{~nm}$, respectively. The resolution for the single-spot exposure scheme was $300 \mathrm{~nm}$. It is apparent from the plots that an order-of-magnitude reduction in job time can be achieved through single-spot exposure for an overall beam-spot size of over $200 \mathrm{~nm}$. The job time for a singlespot exposure scheme decreases as the spot size increases. This is due to a greater total current flowing into the spot with increasing spot size (for example, $2.89 \mathrm{nA}$ for $56 \mathrm{~nm}$ spot and $44.4 \mathrm{nA}$ for a $224 \mathrm{~nm}$ spot), hence requiring reduced exposure time for the same dose.

With all the advantages mentioned above, the technique described is potentially very useful for complex and/or largearea $\mathrm{PhC}$ pattern generation. However, drawbacks have also been encountered. In the single-spot exposure scheme, only square lattice $\mathrm{PhCs}$ with circular holes could be written. In earlier work, non-circular holes have been preferably used in the fabrication of $\mathrm{PhCs}$ with high void-filling ratio (percentage of void area coverage) and small period in order to increase the thickness of walls between adjacent holes [18]. Further investigation is required to check whether our scheme is suitable for small-period $\mathrm{PhCs}$ with high filling-ratio. There is also a limitation on the available resolution and spot size, which are machine dependent. The resolution available from the electron-beam machine used in this experiment, a Leica EBPG5, is continuously variable from 0.005 to $0.3125 \mu \mathrm{m}$, which means that periods over $0.3125 \mu \mathrm{m}$ cannot be written by this scheme on that machine. The available spot sizes are also limited to certain discrete values. While the size of the holes can be controlled easily by changing the dose, as will be shown in the next section, the range of the available resolution 
Electron-beam writing of photonic crystal patterns using a large beam-spot diameter

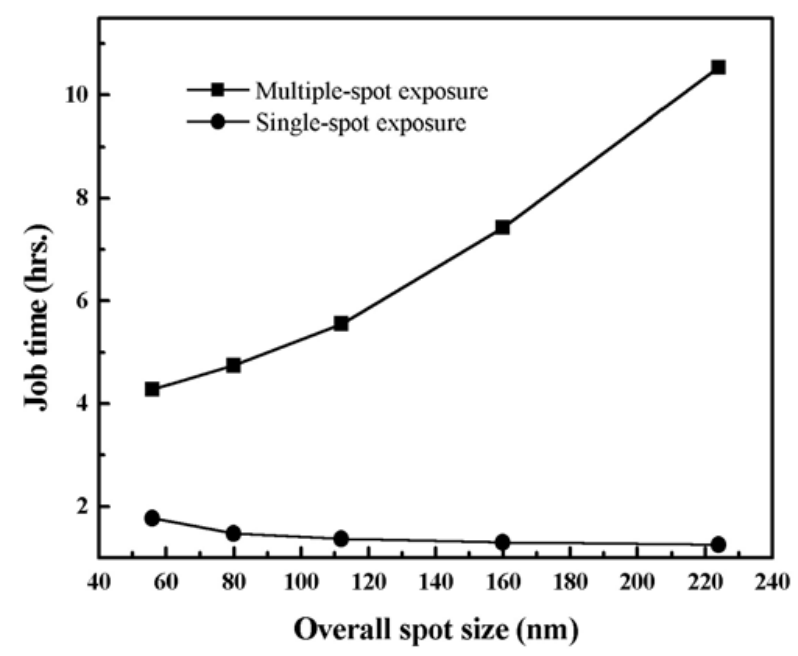

Figure 6. Plots of estimated job times for beam-writing of $\mathrm{PhC}$ patterns with a period of $0.3 \mu \mathrm{m}$ in an area of $500 \times 500 \mu \mathrm{m}^{2}$. The dose was kept constant at $30 \mu \mathrm{C} \mathrm{cm}^{-2}$. The resolution and the spot size for multiple-spot exposure scheme were 5 and $12 \mathrm{~nm}$, respectively. The resolution for the single-spot exposure scheme was $300 \mathrm{~nm}$. The job time for a single-spot exposure scheme decreases as the spot size increases. This is due to a greater total current flowing into the spot with increasing spot size, hence requiring reduced exposure time for the same dose.

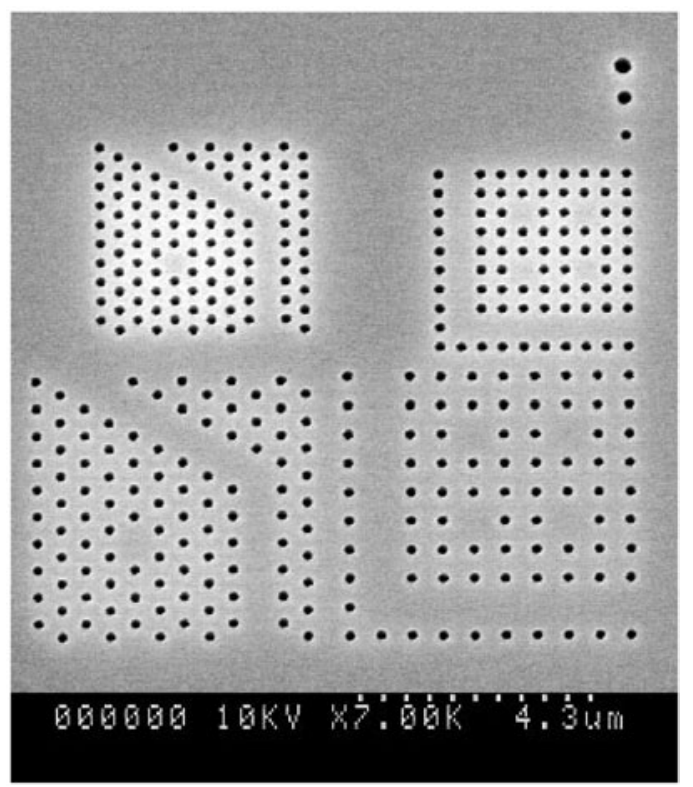

Figure 7. SEM image of test patterns generated by four-spot exposure scheme. PhCs with 400 and $600 \mathrm{~nm}$ periods in both a square lattice and a triangular lattice have been written. The three holes at the top right correspond to the patterns written using $2 \times 2$, $3 \times 3$ and $4 \times 4$ pixels.

cannot be extended without a system-level modification of the Beam-writer.

\section{Four-spot exposure scheme}

Having seen the limitations of the single-spot exposure scheme, a variation of the technique using four spots has been developed which enables the writing of PhCs, with, in

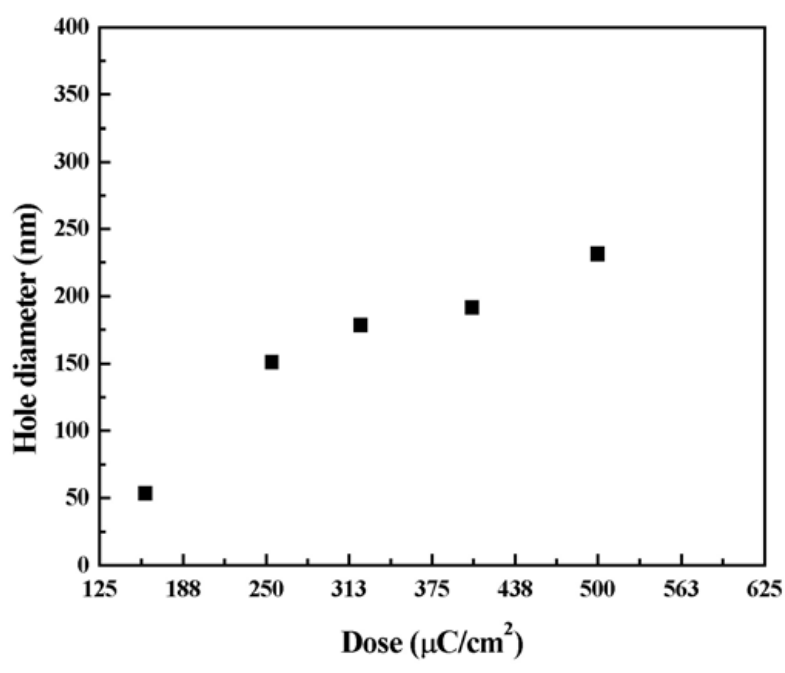

Figure 8. The diameter of holes for the patterns written by the four-spot exposure scheme as a function of dose. The beam diameter was $160 \mathrm{~nm}$ and the resolution was $40 \mathrm{~nm}$.

principle, any period and lattice type. In the four-spot exposure scheme, a smaller resolution is used together with a large spot size comparable to the size of holes to be written. A square consisting of four pixels is used to define a single hole at the design stage. As the size of the electron-beam spot is much larger than the resolution, the four electron-beam spots centred on each pixel will overlap almost completely. The degree of overlap increases as the resolution becomes smaller and the beam size increases. This technique is an improvement on the conventional method in terms of job time as it uses only four pixels to define a single hole and it is better than the single-spot method in terms of flexibility, since it is capable of writing $\mathrm{PhC}$ patterns with any lattice type and period.

An exposure test has been carried out on a sample prepared as described above. Using a $160 \mathrm{~nm}$ beam diameter and a $40 \mathrm{~nm}$ resolution, $\mathrm{PhC}$ patterns with periods of 400 and $600 \mathrm{~nm}$ were written, with both a square lattice and a triangular lattice. Figure 7 shows the SEM image of the developed patterns in UV3 resist. The nominal dose was $406 \mu \mathrm{C} \mathrm{cm}^{-2}$ and the resulting holes had a diameter of $200 \mathrm{~nm}$. The size of the holes can readily be changed by using different pixel sizes, as shown at the top right corner of figure 7 . The three holes, from the bottom upwards, correspond to $2 \times 2,3 \times 3,4 \times 4$ exposed pixels. Another way of controlling hole size is by using different doses. The resultant hole size as a function of dose is plotted in figure 8 . A nominal dose of about $281 \mu \mathrm{C} \mathrm{cm}^{-2}$ results in a hole diameter almost the same as the beam diameter. The clearing dose for four-spot exposure scheme was $156 \mu \mathrm{C} \mathrm{cm}^{-2}$, which seems significantly greater than the one for a conventional exposure scheme $\left(\sim 30 \mu \mathrm{C} \mathrm{cm}^{-2}\right)$. This is a result of a beam diameter four times bigger than the resolution. If a single spot of beam is approximated by a square with a side equal to the beam diameter $d$ and the average local dose for a single spot of beam is defined as $D_{\text {single }}$, the nominal dose $D$ can be expressed as

$$
D=D_{\text {single }}\left(\frac{d}{w}\right)^{2},
$$


where $w$ stands for the resolution. For $D=156 \mu \mathrm{C} \mathrm{cm}^{-2}$ and $d / w=4, D_{\text {single }}$ equals $9.75 \mu \mathrm{C} \mathrm{cm}^{-2}$. If four nearoverlapping spots are used for the definition of a single hole, the resultant dose for the hole becomes $39 \mu \mathrm{C} \mathrm{cm}^{-2}$, which is close to the clearing dose for a conventional exposure scheme.

\section{Conclusions}

Improved electron-beam writing schemes for the generation of complex PhCs have been demonstrated. By using an electronbeam spot size comparable to the size of holes to be written, a significant reduction of writing time has been achieved. It has been shown experimentally that the holes written in this way maintain good sidewall verticality and circularity. The singlespot exposure scheme described has proved to be especially suitable for the generation of square-lattice $\mathrm{PhCs}$ with a period less than $0.3125 \mu \mathrm{m}$. By modifying the operation of the Beamwriter at the system level (control software), the benefit of this scheme could be fully exploited. Compared to the conventional direct-write method, the design of PhC elements is much easier and an order-of-magnitude reduction in job time can be achieved with the single-spot exposure scheme. For $\mathrm{PhC}$ patterns with other lattice configurations, or square lattices with periods over $0.3125 \mu \mathrm{m}$, we have shown that the four-spot exposure scheme described here could serve as an alternative to the conventional method. By using either of the techniques developed here, we believe that cost-effective and good quality $\mathrm{PhC}$ patterns can be generated with relative ease and greater efficiency. However, we recognize that there is still a need for the fine-spot approach in generating more complex (general) hole shapes and the interferometric approach in massproduction of identical patterns.

By combining either of the two approaches above with our techniques, we believe that an improved scheme for the generation of $\mathrm{PhC}$ patterns can be achieved.

\section{Acknowledgments}

This work was supported in part by the European Community through the PICCO project and by the EPSRC under grant no GR/L95212.

\section{References}

[1] Krauss T F, De La Rue R M and Brand S 1996 Nature 383699

[2] Imada M, Chutinan A, Noda S and Mochizuki M 2002 Phys. Rev. B 65195306

[3] Cao J R, Lee P, Choi S, Shafiiha R, Choi S, O'Brien J D and Dapkus P D 2002 J. Vac. Sci. Technol. B 20618

[4] Chutinan A, Okano M and Noda S 2002 Appl. Phys. Lett. 80 1698

[5] Smith C J M, Benisty H, Olivier S, Rattier M, Weisbuch C, Krauss T F, De La Rue R M, Houdré R and Oesterle U 2001 Appl. Phys. Lett. 781487

[6] Smith C J M, De La Rue R M, Rattier M, Olivier S, Benisty H, Weisbuch C, Krauss T F, Houdré R and Oesterle U 2001 Appl. Phys. Lett. 781487

[7] Divliansky I B, Shishido A, Khoo I, Mayer T S, Pena D, Nishimura S, Keating C D and Mallouk T E 2001 Appl. Phys. Lett. 793392

[8] Campbell M, Sharp D, Harrison M, Denning R and Turberfield A 2000 Nature 40453

[9] Berger V, Gauthier-Lafaye O and Costard E 1997 Electron. Lett. 33425

[10] Bogaerts W, Taillaert D, Baets R, Wiaux V and Beckx S 2002 Technical Digest of Integrated Photonics Research (Vancouver, Canada, July 2002)

[11] Kim J and Lee S 2002 J. Appl. Phys. 921185

[12] Ryu H, Kim S, Park H, Hwang J, Lee Y and Kim J 2002 Appl. Phys. Lett. 803883

[13] Moreno E, Erni D and Hafner C 2002 Phys. Rev. B 65155120

[14] Chutinan A and Noda S 2000 Phys. Rev. B 624488

[15] Qiu M and He S 2000 J. Opt. Soc. Am. B 171027

[16] Zijlstra T, van der Drift E, de Dood M J A, Snoeks E and Polman A 1999 J. Vac. Sci. Technol. B 172734

[17] Wendt J R, Vawter G A, Gourley P L, Brennan T M and Hammons B E 1993 J. Vac. Sci. Technol. B 112637

[18] Krauss T, Song Y P, Thoms S, Wilkinson C D W and De La Rue R M 1994 Electron. Lett. 301444 